\title{
Paternidad adolescente ¿Factor de riesgo o de resiliencia?
}

\author{
Denise Benatuil *
}

\section{Resumen}

El estudio del embarazo adolescente ha estado históricamente centrado en la madre, el enfoque preponderante ha sido aquel que considera la maternidad en esta etapa del ciclo vital como un factor de riesgo. Recién en estos años han comenzado a aparecer algunos estudios que enfocan la problemática del embarazo adolescente centrándose en la figura del padre y proponiendo un enfoque salugénico, a partir de la introducción de conceptos tales como el de Resiliencia.

El presente trabajo es de carácter teórico, está realizado partir de datos secundarios. Su objetivo es la compilación de estudios e información sobre la temática de la paternidad adolescente desde un enfoque poco explorado, evaluando los factores de riesgo y resiliencia. Se relevan distintos estudios realizados con jóvenes latinoamericanos.

Asimismo se analizan las posibilidades de acceso de los jóvenes al sistema de salud, el conocimiento de métodos anticonceptivos y la participación en programas de salud reproductiva. Para finalizar se plantea la importancia de incluir a los varones en todo el proceso de procreación y crianza del niño.

Palabras Claves: paternidad- adolescentes- resiliencia.

\section{Abstract}

The study of the adolescent pregnancy has been historically centered in the mother, the preponderant focus has been considers the maternity in this stage of the vital cycle as a factor of risk. Nowadays, have begun some studies that focus the problem of the adolescent pregnancy being centered in the father's figure to appear and proposing a healthy focus, starting from the introduction of such concepts like Resilience.

The present article, is a theoretical work, it is carried out to leave of secondary data. The objective is the compilation of studies and information on the subject of adolescent fatherhood from a less explored focus, considering the factors of risk and resilience. Different studies are raised with Latin American youths.

Lic. en Psicología. Becaria de Doctorado. Adjunta a cargo de las materias Práctica e Integración profesional. Universidad de Palermo. JTP Didáctica Especial de la Psicología UBA.

Correspondencia: Mario Bravo 1259. Ciudad Autónoma de Buenos Aires.

E mail: dbenatuil@iname.com 
Psicodebate 5. Psicología, Cultura y Sociedad

Also are analyzed the access possibilities to the sanitary system from the youths, the knowledge of birth-control methods and the participation in programs of reproductive health. It outlines the importance of including the males in the whole process of procreation and the boy's upbringing.

Key words: paternity - adolescents - resilience. 


\section{Introducción}

En nuestra cultura, circulan estereotipos en cuanto al embarazo adolescente: los varones cuando son adolescentes no pueden ni quieren hacerse cargo de la paternidad, el problema se resuelve mediante la anticoncepción, el embarazo en esta etapa del ciclo vital tiene lugar solo en un sector de la población.

¿Cómo perciben los adolescentes varones la paternidad? ¿Bajo qué circunstancias toman parte en el embarazo? ¿Cuáles son las temáticas que les preocupan? ¿Qué consecuencias tiene la paternidad para un adolescente?

En las últimas décadas ha habido importantes cambios en cuanto a la edad de inicio en la reproducción, retrasándose en las clases sociales medias y altas (favorecido por el acceso de la mujer a niveles más elevados de formación y jornadas más extensas de trabajo), y adelantándose en las clases menos pudientes por una dificultad de acceso a proyectos tales como la educación y el trabajo. Estas modificaciones no implican, como muchas veces se ha creído, que el embarazo adolescente sea no deseado, de riesgo, que se da en chicas solteras y de clases populares.

En nuestro país más del 30\% de los adolescentes de entre 13 y 17 años no concurren a la escuela, la mayoría está trabajando o buscando trabajo. Muchas veces se ven desalentados por la falta de competencias para acceder a un puesto y despliegan a partir de allí una gran variedad de respuestas a su frustración, que muchas veces se expresan en conductas de riesgo para su supervivencia (Konterllnik, 1997). En este contexto, la posibilidad de acceder a la paternidad, como proyecto propio, puede llegar a ser una salida salugénica.

Para poder analizar esta temática y formular algunas posibles respuestas a las preguntas planteadas, en necesario primeramente definir los términos centrales.

La palabra adolescencia viene del latin adolescere que significa crecer hasta la madurez. Por tradición esta etapa se considera un preludio y una preparación para la adultez, un período vital de transición entre la inmadurez y la madurez. Aunque en general se considera que la adolescencia comienza con la pubertad, la considerable variación individual en el momento de la aparición de estos cambios físicos hace muy difícil definirla en términos de edad cronológica (Gross, 1998).

Habitualmente se definía la adolescencia como un período de turbulencia y crisis, creyendo que estas características eran propias de la discrepancia entre la madurez sexual y la inmadurez mental. Actualmente se sabe que esta discrepancia no puede generalizase y solo se dan en algunas sociedades. Por ello la adolescencia debe entenderse como un fenómeno cultural (Stone y Church, 1995). Los adultos y los pares definen los roles que debe desempeñar el adolescente.

Padre proviene del Latín pater: varón o macho que ha engendrado. La Paternidad: proceso psicoafectivo por el cual un hombre realiza tareas en lo concerniente a concebir, proteger, aprovisionar y criar a cada uno de sus hijos; sus funciones se alejan de lo instintivo (Oiberman, 2003). Ser padre es contribuir a la procreación, es tener el comportamiento, el rol y las funciones pero también ser proclamado padre por un niño 
Psicodebate 5. Psicología, Cultura y Sociedad

que afirma su mandato filial en la transmisión (Lebovici, 1995). Ser madre o ser padre no es un mero hecho biológico, implica un reconocimiento simbólico, una filiación de lazos afectivos, con deseos, ideales y deberes.

Comportamientos de riesgo son aquellos que de modo habitual, individuales o colectivos, ponían en juego la seguridad social y/o amenazen la vida de otros (Casullo, 1998). Puede pensarse que los comportamientos de riesgo durante la adolescencia tienen un lugar funcional para lograr la anticipación de experiencias de fracaso, aprender afrontar situaciones de ansiedad y frustración, cuestionar las normas y valores vigentes, lograr autonomía de los padres, lograr la estructuración del self, afirmar y consolidar los procesos de maduración (Jessor, 1991).

Factor de riesgo es la característica o cualidad de una persona o comunidad que va asociada a una mayor probabilidad de daño a la salud (Suárez Ojeda y Kauskopf, 1995).

El presente trabajo posee un enfoque salugénico, por ello se considera de gran relevancia comenzar por la definición de salud reproductiva y continuar con temas vinculados con la salud como la prevención, la resiliencia y el acceso a los programas de salud reproductiva.

\section{Desarrollo}

Las Naciones Unidas (1997) definen la salud reproductiva como el estado general de bienestar físico, mental y social, en todos los aspectos relacionados con el sistema reproductivo, sus funciones y procesos. Entraña la capacidad de disfrutar de una vida sexual satisfactoria y sin riesgos, así como la libertad para procrear o decidir no hacerlo.

Históricamente se ha sostenido un enfoque de la salud reproductiva centrado en la mujer; esta postura ha estado justificada de diversos modos: las mujeres tienen más riesgos de salud asociados con la reproducción que los hombres, los hombres no quieren responsabilizarse. Debido a que son las mujeres quienes cargan con los embarazos, a los hombres rara vez se les pregunta sobre su propia conducta reproductiva; como consecuencia es difícil obtener datos sobre el porcentaje de adolescentes varones y hombres jóvenes que se convierten en padres.

\section{La salud reproductiva y el acceso al sistema de salud}

Actualmente comienza a reconocerse la necesidad de aumentar la responsabilidad de los hombres en lo que concierne a las consecuencias para sus parejas de sus acciones sexuales y su compromiso para la crianza del futuro niño. La falta de inclusión de los varones ha estado dada por diferentes razones y diferentes actores sociales.

Es necesario incluir a los varones en los programas y la asistencia de salud ya que a pesar de tener niveles más elevados de morbilidad y conductas de riesgo que las mujeres; son más reticentes a consultar con un profesional como así también a autopercibir su sintomatología y/o malestar (Wasser y Benatuil). El género masculino es más vulnerable al riesgo debido a patrones aprendidos como la dificultad para expresar emociones y una mayor resistencia al dolor. 
La adolescencia es una etapa del desarrollo en la que los varones están buscando y formando su identidad masculina, establecer su identidad es un proceso de gran importancia y parte de ese proceso es aceptar su sexualidad (Simposio sobre la participación masculina, 1998). Es la etapa de los ritos de iniciación que permiten a un varón ser hombre (Muñoz Chacon, 2001). Un mayor énfasis en los derechos masculinos llevaría a estrategias para crear las condiciones en que los hombres queden habilitados para asumir más responsabilidades. Es innegable que los hombres están implicados en la salud reproductiva de sus parejas, lo que debería modificarse es la forma en la que participan. (Lundgren, 2000).

\section{Conocimientos sobre anticoncepción}

A menudo los jóvenes se sienten invulnerables a los riesgos, esto lleva a que disminuyan su control sobre las cosas que eligen para sí mismos (Cáceres et.al., 1997; Burak, 1997). El grado de madurez cognitiva de un adolescente, el consumo de alcohol y drogas, la falta de habilidades comunicacionales por falta de experiencia, la baja autoestima, las expectativas sociales y los tabúes culturales relacionados con la sexualidad pueden limitar su habilidad de planear sus relaciones sexuales, articular claramente sus valores personales, negociar con una pareja, y obtener contraceptivos y condones (Clark, Zabin y Hardy, 1984; Barker, 1996).

Existen muchas barreras y normas culturales en América Latina que impiden a los adolescentes varones mantener su salud reproductiva y sexual. Muchos hombres se enfrentan a expectativas sociales que los suponen conocedores y experimentados en temas de sexualidad, por lo que no deberían tener la necesidad de buscar información relativa a su salud sexual (Barker, 1996). Al buscar información temen quedar expuestos, sintiéndose ignorantes. A menudo, en comunidades latinas, se marca la conversión de los varones en adultos fomentando la actividad y experimentación sexual, en estos casos también se suele atribuir a las mujeres la responsabilidad por la anticoncepción (De La Vega, 1990). También muchas culturas dictaminan que la contracepción es responsabilidad de las mujeres y desaniman la participación masculina en la toma de decisiones relativas a la contracepción (De La Vega, op.cit.1990).

Se han realizado investigaciones sobre el uso de anticonceptivos. En general, el método usado más frecuentemente en la primera relación sexual prematrimonial en América Latina fue el condón, seguido por contraceptivos orales y después el método del ritmo. Sin embargo, en Ciudad de México y Santiago de Chile el método más usado para la primera relación sexual fue el método del ritmo, en Sao Paulo la interrupción del coito y en Ciudad de Guatemala la píldora (OPS, 1997).

Muchos estudios coinciden en el uso de condones sólo se da con parejas casuales porque está asociado a la prostitución y las enfermedades de transmisión sexual (Cáseres, op.cit. 1997). Si bien en el primer coito la mayoría manifiesta usar condón o el método del ritmo, pocos jóvenes pudieron identificar el período más fértil en el ciclo femenino, en un menor número de casos utilizan anticonceptivos orales (Morris, 1988). 
Psicodebate 5. Psicología, Cultura y Sociedad

Un estudio realizado en Jamaica, revela que para algunos adolescentes es más fácil acceder a un aborto que a métodos anticonceptivos (Boland, 1997).

Las bajas tasas de uso de anticonceptivos, particularmente el condón, pone a los adolescentes varones y hombres jóvenes en riesgo de contraer enfermedades de transmisión sexual y de embarazos no deseados. Esto, combinado con el hecho de que la primera experiencia sexual de los hombres es a menudo con una mujer mayor y de más experiencia o con una prostituta, tiene consecuencias muy serias. En el Caribe, un estudio reportó que la mitad de los jóvenes sexualmente activos no usaron contraceptivos en su última relación sexual (OPS, 1997). Marsiglio encontró que los hombres jóvenes con actitudes tradicionales respecto a los roles de género tienden a tomar más riesgos en su comportamiento sexual y contraceptivo (1993).

Diferentes estudios dan cuenta de que los adolescentes, tanto varones como mujeres expresan que a menudo les da miedo hablar de sexo y contracepción con su pareja y necesitan orientación sobre cómo comunicar sus preocupaciones. Muchas de las adolescentes embarazadas dijeron que preferían arriesgarse a quedar embarazadas antes que aparecer como conocedoras de asuntos de sexo y contracepción.

Fischer (1990) sostiene que es frecuente que los adolescentes tengan información poco relevante, errada o inadecuada sobre concepción, anticoncepción y enfermedades de trasmisión sexual.

A pesar de estar en el siglo XXI, todavía la sexualidad y la anticoncepciones siguen siendo temas tabú.

\section{Factores de Riesgo}

Distintos estudios sistematizan los factores que favorecen los comportamientos sexuales riesgosos: niveles educacionales más bajos, ambientes peligrosos e inadecuados, falta de apoyo de los padres, pocas relaciones positivas con sus padres u otros adultos varones que pueden servir como modelos, socialización en un grupo de pares con experiencia sexual y débil compromiso religioso (Kiragu y Zabin 1993; Pick et al.,1991). En algunos trabajos aparecen asociados a las conductas sexuales riesgosas, la violencia, los conflictos intrafamiliares, baja autoestima, abandono escolar temprano y falta de control externo (Burak, 1998).

En el caso de las mujeres, hay gran cantidad de trabajos que asocian el embarazo con la falta de espacio propio, de trabajo y de actividad educativa. Estas jóvenes encuentran la maternidad como único proyecto de vida (Greco, 2001).

Se ha demostrado que para los jóvenes en general, el uso de las drogas y el alcohol está asociado a un riesgo más alto de enfermedades de transmisión sexual y embarazos. El sexo frecuente bajo la influencia de drogas y alcohol está asociado a problemas de salud reproductiva, tanto enfermedades de transmisión sexual como embarazos no deseados (Cáceres et. al, 1997).

En un estudio de adolescentes de edad escolar en Kenya se halló que los comportamientos sexuales riesgosos estaban relacionados con la socialización en un 
grupo de pares con experiencia sexual, un compromiso religioso débil, actitudes tolerantes hacia el sexo premarital y un ambiente familiar inestable (Kiragu y Zabin, 1993). Los hombres jóvenes que se involucran en comportamientos riesgosos a menudo tienen pocas relaciones positivas con sus padres u otros adultos varones que pueden servir como modelos y mentores (OPS, 1997). En México, Pick y Palos (1995) encontraron que la comunicación entre los padres e hijos respecto a la sexualidad tiene una influencia significativa en el inicio de la vida sexual de los niños, así como también en los embarazos adolescentes y el uso de contraceptivos. Otros factores citados en la literatura que están asociados a las conductas sexuales riesgosas incluyen la violencia y los conflictos intrafamiliares, una baja autoestima, abandono escolar temprano y falta de control externo (Burak, 1998).

Los estudios coinciden en que la edad no es el principal factor de riesgo para los embarazos adolescentes, en cambio se considera tiene gran influencia el conjunto de circunstancias económicas, afectivas, familiares y culturales.

El machismo se presenta como un factor predictor del embarazo en la adolescencia; las relaciones son absolutamente dominantes. El padre, padrastro y/o hermanos muestran una actitud celosa con respecto al trato de la adolescente cuando se relaciona con otras personas, especialmente si son hombres. Las relaciones autoritarias predisponen una conducta de rebeldía por parte de la adolescente. Esto puede significar que ella termine por apegarse a sus pares con más fuerza, y que las relaciones de noviazgo a edades tempranas cobren importancia en el comportamiento sexual de la adolescente, confundiendo el acto sexual con el afecto.

\section{Resiliencia}

Las investigaciones sobre salud reproductiva de los adolescentes han tendido a centrarse en la identificación de factores de riesgo, dejando de lado los factores que protegen a los jóvenes de las influencias negativas. Se llama resilentes a aquellos individuos que a pesar de los factores de riesgo y adversidad asociados a su medio ambiente desarrollan, mediante un proceso dinámico, comportamientos adaptativos (Luthar et. al. 2000, citado por Merillo y Suárez Ojeda).

Las características y factores protectores comúnmente identificados en los individuos resilentes son: autoestima, capacidad de autocontrol, ausencia de discordia matrimonial, cohesión familiar, buena relación con por lo menos uno de los padres, nivel satisfactorio de educación, buena nutrición, buena sanidad ambiental e higiene personal, empleo, valores y normas religiosas, fuertes redes familiares y de amigos, y normas políticas y legislativas que favorecen a los niños (Burak, op.cit. 1998; OPS, 1997).

Grotberg (1996) caracteriza la resiliencia como aquello que permite afrontar la adversidad, superarla y salir fortalecido como así también transformado por la experiencia. Rutter (1993) sostiene que es un conjunto de procesos sociales e intrapsíquicos que posibilitan tener una vida sana en un medio insano.

Distintas investigaciones dan cuenta de que los factores protectores pueden actuar como una verdadera defensa para favorecer el desarrollo de los seres humanos (Prieto 
Psicodebate 5. Psicología, Cultura y Sociedad

et. al, 2001). La resiliencia disminuye la vulnerabilidad al stress y la posibilidad de verse afectado por eventos negativos, paralelamente se incrementa la predisposición a compensar la frustración. El enfrentamiento gradual de la adversidad fortalece las posibilidades de aprendizaje de respuestas.

Generalmente la tendencia ha sido preocuparse por el embarazo adolescente, olvidando que algunos de esos embarazos pueden ser deseados, incluso aunque no sean intencionados. Los adolescentes varones y hombres jóvenes pueden juzgarse a sí mismos como listos para formar una familia siendo el embarazo un factor protector que mejore su autoestima, los haga sentir como hombres "de verdad" y aumente el control sobre su pareja. (Lundgren, 2000).

Es probable que los hombres jóvenes en desventaja socioeconómica vean la paternidad como una fuente de autoestima y crean que ser padres puede reforzar su sentido de masculinidad (Sonenstein, Pleck y Ku, 1991). Un posible beneficio a menudo descuidado del compromiso de un padre es el potencial inmenso para promover mayor responsabilidad, comprensión, madurez y compromiso con la crianza entre los hombres.

Asimismo hay que considerar que la paternidad temprana también puede tener consecuencias negativas para los hombres jóvenes. La joven pareja puede verse atrapada en una vida de pobreza con una familia grande y un matrimonio infeliz, los nuevos roles pueden implicar la necesidad de interrupción de la educación. Más aún, el estrés de la situación puede manifestarse en abuso de sustancias, violencia y otros comportamientos negativos para su salud y la de su familia (Consejo Nacional de Población, 1995).

El temor a no poder asumir el papel de padre y las consecuencias que esto acarrea para la autoestima, pueden llevar a la comprensión de la inestabilidad en las parejas jóvenes, esta situación se agrava por el nulo papel asignado al hombre durante el embarazo, nacimiento y crianza de los hijos. Muchas veces se suma la descalificación de otros hombres (familiares y /o grupo de pares). No poder cumplir con las funciones asignadas socialmente a la paternidad lleva a una crisis de identidad con riesgos como el abandono de la pareja y los hijos o la generación de conductas violentas (Muñoz Chacon, 2001).

\section{Aceptación de la paternidad}

La reacción de los hombres jóvenes y adolescentes a un embarazo no intencionado varía desde la negación, el deseo de abortar, la aceptación de su responsabilidad hasta la participación total como padre. Sus actitudes influencian las acciones de las mujeres jóvenes, tanto directa como indirectamente. Distintas investigaciones dan cuenta de que la primer persona a la que las mujeres piden consejos es a su pareja. La actitud del padre hacia el embarazo usualmente es un factor clave en la decisión de una muchacha sobre cómo manejar un embarazo no intencionado. La imagen del joven irresponsable que abandona a la novia embarazada, contradice el apoyo dado por padres jóvenes (Papalia y Wendkos, 1997). 
En un trabajo realizado en Chile, el grupo de padres adolescentes sostuvo en su mayoría $(83,5 \%)$ que apoyarían a su pareja en lo afectivo y en lo económico, la mitad del grupo sostuvo que reaccionó feliz ante la noticia que iba a ser padre, un grupo más pequeño se preocupó mucho ante esta responsabilidad con una reacción de susto. La mayoría de los varones indicó que la noticia del embarazo provocó cambios personales importantes en sus vidas, como: ser más responsable, portarse mejor, tener más conflictos (González et.al, s/f.).

El embarazo adolescente para la mujer habitualmente tiene efectos positivos, promoviendo el acercamiento de la futura madre a la familia extendida y a los amigos. El embarazo suele ser evaluado por la joven como dando sentido a su vida, la posibilidad de ser alguien y la esperanza de tener un futuro mejor (Piñero, 2001).

Desde el discurso social se suele sostener que los varones jóvenes no se involucran en el embarazo de sus parejas, sin embargo hay distintos factores que influyen en esta situación. En muchos contextos los adolescentes varones son educados con la creencia que ellos no son responsables de los embarazos o los niños que resulten de su primera actividad sexual (Barker, 1996). Las instituciones sociales -familia, colegios y sistema de atención de salud- suelen apoyar esta visión, la aceptación varonil de la paternidad a menudo es condicional, contingente a la prueba de paternidad, la calidad de la relación con la madre, las expectativas de los padres y la sociedad, y su habilidad de proveer apoyo económico (Castillo, Dwek y Levy, 1988).

La investigación en América Latina ha encontrado que la situación de empleo de un hombre joven afecta en gran parte el nivel de responsabilidad que acepta por un hijo (Barker y Lowenstein, 1996). En un estudio chileno se encontró que era cinco veces más probable que el padre proporcionara apoyo al niño si tenía empleo (González et.al, op.cit.). En su estudio de responsabilidad procreativa, Marsiglio (1993) encontró que los tres factores relacionados con la aceptación de la responsabilidad por el embarazo son la educación, el barrio y la fuerza de la creencia en roles de género varonil tradicionales.

Muchos adolescente manifiestan que sus propios padres son la fuente más importante de consejos y apoyo respecto de su rol paternal, temiendo desilusionarlos si no actuaban de acuerdo a sus expectativas. También eran susceptibles a la presión social, temiendo dar un mal ejemplo y fallar ante los seres queridos y la comunidad.

Cuando las decisión es no continuar con el embarazo los hombres también tienen un rol central. En América Latina están involucrados en la toma de decisiones sobre el aborto a través del apoyo financiero como así también mediante su ausencia. Los resultados de las Encuestas de Salud Reproductiva de Adultos Jóvenes efectuadas en América Latina encontraron que entre $32 \%$ y $60 \%$ de los varones reportaron que por lo menos una de sus parejas había tenido un aborto (Morris, 1993). En Río de Janeiro y Recife, en Brasil, el porcentaje fue de 59\%. De los hombres que reportaron que su pareja había tenido un aborto, el $24 \%$ dijo que había participado en el proceso de toma de decisión conducente al aborto. 
Psicodebate 5. Psicología, Cultura y Sociedad

Para muchos, las diferencias en el comportamiento sexual de ambos géneros se origina en la doble moral sexual que impera en nuestra sociedad. Pero los jóvenes adoptan una actitud crítica frente a las desigualdades de género, considerando que tal situación tiene también desventajas (Coll, 2001a)

En nuestra cultura no se les enseña a los varones a criar y cuando se convierten en padres se les dice que son irresponsables y que no saben cuidar a los niños. Un resultado importante de varios estudios es que mientras más temprano un hombre se involucra con su hijo, es más probable que se mantenga comprometido con ese niño (Palkovitz, 1985). Un estudio longitudinal de padres adolescentes mexicanos encontró que si el padre estaba presente en el nacimiento del hijo, aceptando su responsabilidad, era más probable que se mantuviera.

Greenberg y Morris (1982) realizan un estudio sobre el contacto temprano entre el padre y el bebé a partir del concepto de engrossement, llegando a la conclusión de que aquellos padres que habían participado en el parto manifestaban tener un sentimiento paterno alto, estar conformes con el sexo del bebé, podían distinguir su hijo de los otros bebés, querían compartir con sus parejas el cuidado del bebé y estaban dispuestos a tomar al bebé en brazos. Vivian la paternidad con extrema exaltación y muchos dieron cuenta de un aumento en la autoestima a partir del nacimiento.

La escala de observación paterna (Oiberman, 1999), aplicada a 60 padres argentinos de diversas edades, permitió verificar que en general son los padres más jóvenes (menores de 20 años) y primerizos, quienes tuvieron mayores conductas de acercamiento (mirar, acariciar, sostener) a sus hijos recién nacidos.

Se desconoce el porcentaje de hombres jóvenes que aceptan su responsabilidad frente a un embarazo no planificado. Tampoco se sabe lo que esta responsabilidad acarrea o cuando tiempo dura.

Son escasos los servicios de asistencia que incorporan al padre en la atención, esta actitud suele aparecer naturalizada, convocando a la madre de la menor para que se haga cargo de la adolescente y de su hijo. Se ha visto que muchas veces los adolescentes varones se encuentran comprometidos con el embarazo y son sus padres quienes los incitan a desistir de la decisión de hacerse cargo del bebé.

Distintos estudios realizados en América Latina dan cuenta de que cuando un padre no puede cumplir con el rol de proveedor de sus hijos, su asociación con la madre se debilita y su autoridad y relación con los hijos se hace tenue, sienten que no pueden hacer ninguna otra contribución a la familia y los hijos (Barker y Loewenstein, 1996; Muñoz Chacon, 2001).

En los padres jóvenes la Responsabilidad aparece vinculada al rol de protectorproveedor, permitiendo el proceso de independización que le permita conformar su propio hogar y enfrentar las necesidades diarias de su familia. Pero los jóvenes deben enfrentarse a la falta de trabajo y los trabajos precarios, lo que dificulta la posibilidad de independización (Muñoz Chacon, 2001). 


\section{Programas de Salud Reproductiva}

En América Latina un grupo importante de adolescentes sexualmente activos, carecen de acceso a educación, información, servicios de salud sexual y reproductiva, como consecuencia quedan impedidos para ejercer sus derechos reproductivos. Las marcadas diferencias entre los adolescentes de esta región y los de países desarrollados, no están en las edades en las que comienzan a ser sexualmente activos, sino en la edad en que comienzan su reproducción (Villa y Rodríguez, 2001).

Como ya se ha dicho, la información que tienen los adolescentes varones y hombres jóvenes sobre sexualidad, fertilidad y contracepción suele ser inadecuada e incorrecta. Generalmente mencionan la falta de conocimiento más a menudo que las mujeres, y dicen que es responsabilidad de la pareja evitar el embarazo. Son muchos los jóvenes que desconocen los métodos anticonceptivos, manejan su salud reproductiva a partir de mitos o del pensamiento mágico.

Existe una necesidad crítica de educación sexual para los varones. Los programas educacionales pueden darle a los jóvenes las habilidades que necesitan para posponer su iniciación sexual, y pueden aumentar el uso de anticonceptivos. Morris (1988) plantea que la combinación de un inicio sexual temprano y la falta de conocimientos sobre fertilidad apuntan a la necesidad de programas efectivos de educación sexual en los colegios de enseñanza básica.

Según algunos estudios, las políticas de prevención del embarazo en la adolescencia han tendido a excluir a los hombres, así como también a la familia, focalizando la atención en las adolescentes. En la distribución de responsabilidades, se asigna la totalidad de la carga de la prevención de los embarazos a las mujeres. Los hombres terminan siendo excluidos de toda o mayor parte de la responsabilidad, y la familia asume el control de las adolescentes, antes que su formación para un ejercicio autónomo y sano de la sexualidad (Núñez Rivas y Rojas Cavaría; 1998).

Dos temas importantes que a menudo se pasan por alto son las habilidades para criar niños y para comunicarse. Algunos hombres pueden no involucrarse en la paternidad porque se sienten inadecuados. Esta es la razón por la cual son importantes los grupos de discusión o de apoyo con padres jóvenes donde los hombres puedan intercambiar experiencias y desarrollar sentimientos positivos sobre sus habilidades como padres (Barker, 1996).

Para realizar programas para mejorar la salud reproductiva de los varones se debe tener en cuenta: 1) la posibilidad de acceso a los lugares donde están los hombres ya sean las escuelas o lugares de trabajo; 2) involucrar a sus pares, padres e instituciones sociales; 3) ayudar a los hombres a encontrar formas para expresarse y trabajar con ellos para modificar ciertas funciones de género; 4) proporcionar servicios especiales a los padres jóvenes y primerizos desde los sistemas de salud y educación.

En nuestro país muchas maternidades no permiten el ingreso o permanencia de los padres en las habitaciones, salas de espera o residencias. En estos casos la exclusión del padre está dada desde lo institucional. 
Psicodebate 5. Psicología, Cultura y Sociedad

Por último un posible beneficio, a menudo descuidado, del compromiso de un padre es el potencial inmenso para promover mayor responsabilidad, comprensión, madurez y compromiso con la crianza entre los hombres. La participación parental tiene importantes beneficios para los niños, algunos estudios dan cuenta de que aquellos niños que se han criado con ambos progenitores tiene un mejor desempeño escolar, desarrollo social y emocional, y su salud y bienestar general es mejor.

\section{Conclusiones}

Del presente trabajo se desprende la gran disociación que existe entre un discurso que identifica el embarazo adolescente como un factor de riesgo, un hecho problemático, presente sólo en las clases populares, no deseado y por otro lado el discurso de los jóvenes que manifiestan que el embarazo posibilita encontrar un lugar a partir de su rol de madre/padre, encontrarle sentido a la vida, incrementar la esperanza de tener un futuro mejor, aumentar su autoestima y la responsabilidad.

A partir de lo desarrollado se debe pensar en la inclusión de los varones en programas de salud reproductiva, involucrando a sus pares, padres e instituciones sociales; ayudando a los hombres a encontrar formas para expresarse y trabajar con ellos; proporcionando servicios especiales a los padres jóvenes y primerizos. Estos programas deberían ir acompañados del cambio en los roles de género tradicionales, propiciado desde los distintos actores sociales y los medios de comunicación.

Las respuestas que pueden darse desde la salud pública requieren estrategias múltiples e integradas como: los estilos de vida individuales, la provisión de los servicios de salud, el contexto comunitario, el entorno y las políticas públicas. Pero además requieren cambios en la provisión de servicios (mejorando la disponibilidad y accesibilidad a los servicios de salud), el en entorno social y político (Touzé, 1999)

Si bien en la mayoría de los países se observa una disminución de la fecundidad en la adolescencia, esto no sucede en la Argentina donde desde los años 70 hasta los 95 ha habido un leve incremento (Guzmán, Contreras y Hakkert, 2001). Estos datos, permiten sugerir la necesidad inminente de afianzar y mejorar los programas de salud reproductiva que están funcionando en nuestro país.

Distintos trabajos han dado cuenta de la relación existente entre la falta de proyecto y el embarazo adolescente. En las clases más pobres, los adolescentes tienen pocas opciones de realización personal por medio del estudio o el trabajo, por lo tanto la paternidad temprana puede transformarse en una de las principales opciones de realización personal y accesos a la vida adulta. Si socialmente se les da lugar, seguramente en un futuro no muy lejano habrán más padres adolescentes que podrán y querrán comprometerse con la crianza de sus hijos. Y en un futuro cercano podremos ver más niños resilientes por haberse criado en un ambiente de respeto, afecto y cariño. 
Paternidad adolescente ¿Factor de riesgo o de resiliencia?

\section{Bibliografía}

Barker, G. (1996) The Misunderstood Gender: Male Involvement in the Family and in Reproductive and Sexual Health in Latin America and the Caribbean. United States: Erickson Institute.

Barker, G. \& Loewenstein, I. (1996) Where the Boys are: Promoting Greater Male Involvement in Sexuality Education: Conclusions from Qualitative Research in Rio De Janeiro. Brasil: Centro De Educação Sexual, CEDUS.

Boland, B. (1997) Dinámica de la población y desarrollo en el Caribe: con especial énfasis en la fecundidad de los adolescentes, la migración internacional, las políticas de población y la planificación del desarrollo. Cuadernos del CEPAL, $N^{\circ} 76$. Santiago de Chile: CEPAL.

Burak, S.D. (1998) Protección, riesgo y vulnerabilidad, Organización Panamericana de la Salud, Junio.

Burak, S.D. (1997). Adolescent's Integral Health: A Conceptual and Epidemiological Framework. PAHO, WHO, Program for Adolescent's Integral Health. Caracas, Venezuela. September.

Caceres, C., Vanoss Marin, B., Hudes, E., Reingold, A., Rosasco, A. (1997) Young People and the Structure of Sexual Risks in Lima. AIDS, 11(1), 67-77.

Castillo, M.; Dwek, L.; Levy, E. Y Cols. (1988) Maternidad adolescente en medios suprivilegiados. Buenos Aires: Humanitas.

Casullo, M.M. (1998) Adolescentes en Riesgo. Buenos Aires: Paidós

Clark, S., Zabin, L. y Hardy, J. (1984) Sex, Contraception and Parenthood: Experience and Attitudes among Urban Black Young Men. Family Planning Perspectives, 16-77.

Coll, A. (2001a) Información para la Comunidad. Los aspectos del genero en el embarazo en la adolescencia. En: http://www.SAGIJ Sociedad Argentina de Ginecología Infanto Juvenil_archivos/SAGIJ Sociedad Argentina de Ginecología Infanto Juvenil.htm

Coll, A. (2001b) Embarazo en la adolescencia ¿Cual es el problema? En: S.D. Burak. Adolescencia y Juventud en América Latina. Costa Rica: CEPAL.

Consejo Nacional de Población, (1995) Estrategia de Capacitación Sobre Salud Sexual y Reproductiva del Adolescente. México: DF. 
Psicodebate 5. Psicología, Cultura y Sociedad

De La Vega, E. (1990). Considerations For Reaching the Latino Population with Sexuality and HIV/AIDS Information and Education. SIECUS Report. 18(3): 1-8.

Fischer J.D. (1990) Understanding and preventing adolescent pregnancy and sexually transmissible disease. En: J. Edwards, R. Tindale, L. Heath y E. Posavac (Eds.) Social influence processes and prevention. Nueva York: Plenum Publishing Co.

González, E.; Toledo, V.; Luengo, X.; Molina, T.; Meneses, R. (s/f.) Paternidad adolescente: Variables familiares e impacto de la paternidad en el padre adolescente. Santiago de Chile: Facultad de Medicina, Universidad de Chile, Centro de Medicina y Desarrollo Integral del Adolescente.

Greco, M.B. (2001) Cuando los/as jóvenes tienen la palabra. El embarazo adolescente: una temática para trabajar en la escuela. Revista Ensayos y Experiencias N38 Año 7 (53-63)

Grotberg, E. (1996) Guía de promoción de la resiliencia en niños para fortalecer el espíritu humano. Informes de trabajo sobre el desarrollo de la infancia $N^{\circ} 18$. Holanda: Fundación Bernard van Leer.

Greenberg y Morris (1982) Engrossment: The newborn's impact upon the father. Stanley Cath, Alan Gurwitt and Munder Ross (eds.) Father and Child Developmental and Clinical perspectives. Boston: Little Brown and Co.

Gross, R. (1998) Psicología la ciencia de la mente y la conducta. México: El Manual Moderno.

Guzmán, J.; Contreras, J. y Hakkert, R. (2001) La situación actual del embarazo en la adolescencia en América Latina y el Caribe. En: S.D. Burak. Adolescencia y Juventud en América Latina. Costa Rica: CEPAL

Jessor, R. (1991) Risk behavior in adolescence: A psychosocial framework for the understanding and action. Journal of Adolescence Health Care, 12, 597-605.

Kiragu, K y Zabin, L. (1993). The Correlates of Premarital Sexual Activity among SchoolAge Adolescents in Kenya. International Family Planning Perspectives, 19:92-97 \& 109.

Konterllnik, I (1997) ¿Por qué la adolescencia?. En: I, Konterllnik y C. Jacinto (comp.) Adolescencia, pobreza, educación y trabajo. Buenos aires: Losada.

Lebovici, S. (1995). Pères et Bèbès. En: A. Oiberman (1999) Padre-Bebé: inicio de una relación. Argentina: Universidad Nacional de La Plata 
Paternidad adolescente ¿Factor de riesgo o de resiliencia?

Lundgren, R. (2000) Protocolos de investigación para el estudio de la salud sexual y reproductiva de los adolescentes varones y hombres jóvenes en América Latina. División de Promoción y Protección de la Salud. Programa de Familia y Población. OPS.

Marsiglio, W. (1993). Adolescent Males' Orientation toward Paternity and Contraception. Family Planning Perspectives, 1, Vol 25:22-31

Merillo, A. y Suárez Ojeda, E. (2001) Resiliencia. Descubriendo las propias fortalezas. Buenos Aires: Paidós.

Morris, L. (1988). Young Adults in Latin America and the Caribbean: Their Sexual Experience and Contraceptive Use. Family Planning Perspectives, 14 (4): 153-158.

Muñoz Chacon, S. (2001) En busca del Pater Familia: construcción de la identidad masculina y paternidad en adolescentes y jóvenes. En: S.D. Burak. Adolescencia y Juventud en América Latina. Costa Rica: CEPAL

Núñez Rivas, N. y Rojas Cavaría, A. (1998) Revisión conceptual y comportamiento del embarazo en la adolescencia en Costa Rica, con énfasis en comunidades urbanas pobres. Revista Costarricense de Salud Pública 7(13):38-54,

Oiberman, A.(1999) Padre-Bebé: inicio de una relación. Argentina: Universidad Nacional de La Plata.

Oiberman, A (2003). Material utilizado en el curso de Doctorado. Marzo de 2003. U. de Palermo.

OPS (1997) Encuesta de Salud de Adolescentes del Caribe. Organización Panamericana de la Salud.

Palkovitz, R. (1985) Fathers birth attendance, early contact, and extenden contact with their newborns a critical review. Child Development, 56.

Papalia, D y Wendkos, O.S (1997) Desarrollo Humano. Con aportaciones para Iberoamérica. $6^{\circ}$ Edición. Colombia: Mc Graw Hill.

Pick De Weiss, S., Atkin, L., Gribble, J. \& Andrade-Palos, P. (1991). Sex, Contraception and Pregnancy among Adolescents in Mexico City. Studies in Family Planning, 22(2):74-82.

Piñero, L. (2001) Pensar y actuar sobre el embarazo adolescente a partir de un discurso popular y científico extremadamente heterogéneo. Revista Ensayos y Experiencias N³8 Año 7 (69-79) 
Psicodebate 5. Psicología, Cultura y Sociedad

Prieto, E.; Ruta, M.; Juárez, L.; Garro, M. (2001) Resiliencia y factores de riesgo en el embarazo adolescente. Claves, XI (19-20); 37-40

Rutter, M. (1993) La resiliencia: consideraciones conceptuales. N.Y: Elsevier Science Publishing Co.

Simposio sobre la participación masculina en la salud sexual y reproductiva (1998). Oxaca, México: Red de Masculinidad. http://www.flacso.cl/masculinidad

Sonenstein, F., Pleck, J., y Ku, L. (1991). Levels of Sexual Activity among Adolescent Males in The United States. Family Planning Perspectives, 23(4), 162-167.

Stone, L.G. y Church, J. (1995) Niñez y adolescencia .19 Edición. Argentina: Lumen Hormé.

Touzé, G. (Comp.) (1999) Prevención del HIV-SIDA en usuarios de Drogas. Buenos aires: Intercambios.

Villa, M. y Rodríguez, J. (2001) Juventud, reproducción y equidad. En: S.D. Burak. Adolescencia y Juventud en América Latina. Costa Rica: CEPAL.

Wasser de Diuk, L y Benatuil, E (2000) Evaluación de Síntomas Psicopatológicos en Adolescentes mediante la técnica SCL- 90 R. (documento no publicado) 